\title{
COMPASS: meson spectroscopy and low-energy meson dynamics
}

\author{
Jan M. Friedrich ${ }^{1, *}$ \\ for the COMPASS Collaboration \\ ${ }^{1}$ Institute for Hadronic Structure and Fundamental Symmetries, \\ Physics Department, Technical University of Munich, Germany
}

\begin{abstract}
Within the broad physics programme of the COMPASS collaboration at CERN SPS, soft reactions of high-energy hadron beams on different nuclear targets are investigated. Aiming at a better understanding of the strong interaction, novel results range from tests of chiral symmetry breaking to properties of diffractively produced meson resonances studied in their multi-particle decays, such as the $a_{1}(1420)$ with unusual properties. The talk will highlight the challenges of the employed experimental techniques, and as well of the analysis methods, that are developed to a large extent along with the analysis of the world's largest data sets in the field.
\end{abstract}

\section{Introduction}

Properties of the pions $\left(\pi^{-}, \pi^{0}, \pi^{+}\right)$and of their excitation spectrum into states of higher mass and spin are important benchmarks in understanding quantum chromodynamics (QCD) of light quarks and gluons. The COMPASS collaboration uses pion beams produced by the CERN Super Proton Synchrotron in order to determine such properties in soft reactions by photon and Pomeron exchange with protons and nuclei.

The pions are identified in the framework of the low-momentum expansion of QCD, chiral perturbation theory (ChPT), as the Goldstone bosons emerging from the spontaneous breaking of chiral symmetry. Pion-pion scattering has been studied by several approaches, e.g. in kaon decays, and successfully described within ChPT. In contrast, for pion-photon interactions even the most fundamental process of Compton scattering has remained a riddle since the leading structure-dependent term, the electromagnetic polarisabilities had been determined from first data in 1983, confirmed by some later experiments, while deviating from other results.Clarifying this subject was one of the initial motivations for the experiments presented in the following. Other pion-photon interactions with more pions in the final state came also into reach and are studied as well. Most importantly, the chiral anomaly describes the threshold behaviour of the reaction $\pi^{-} \gamma \rightarrow \pi^{-} \pi^{0}$ and its determination from the COMPASS data is ongoing.

*e-mail: jan@tum.de. Supported by the German ministry BMBF, the Maier-Leibnitz-Labor der LMU und TU München, and the DFG Cluster of Excellence "Origin and Structure of the Universe". 


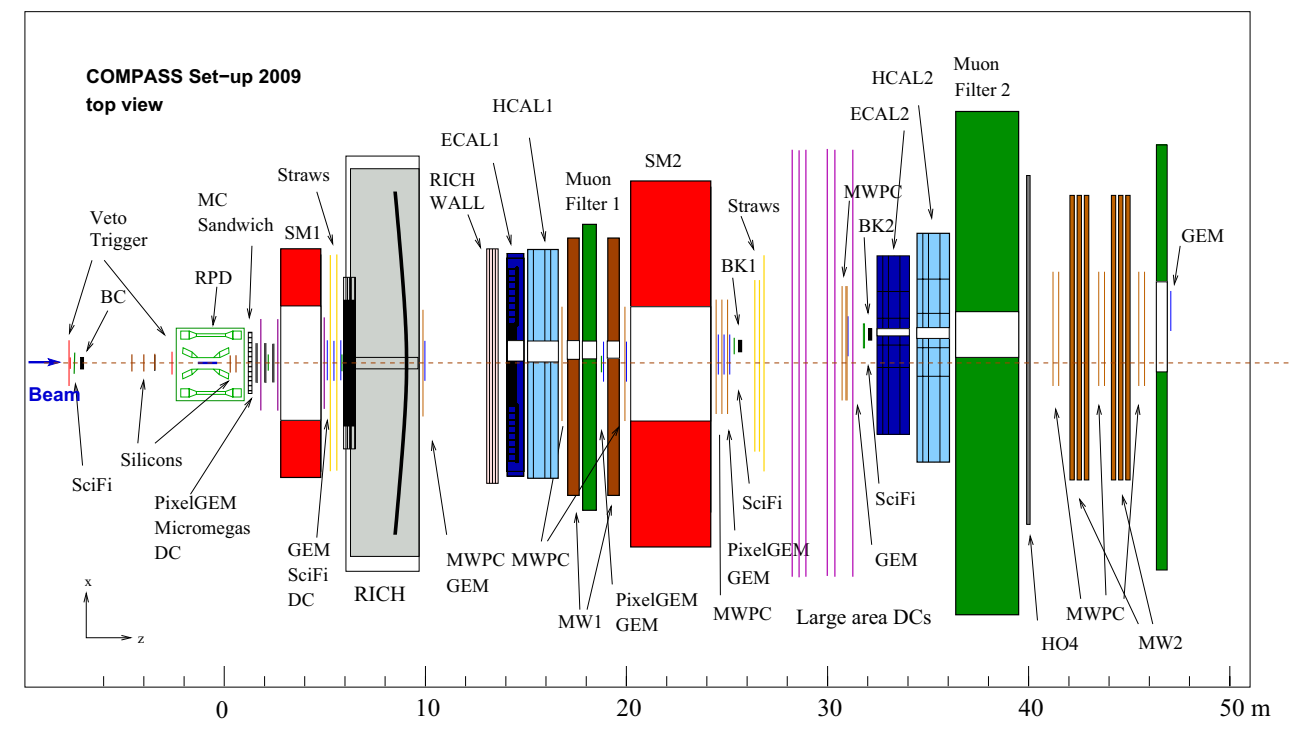

Figure 1. Layout of the COMPASS setup for hadron beam as used in the beam times of 2008 and 2009. A detailed description of the employed detectors is found e.g. in Ref. [1]. The spectrometer magnets SM1 and SM2, surrounded by tracking detectors and followed by the calorimeters, constitute the two stages of the setup.

\section{COMPASS apparatus}

COMPASS deploys secondary hadron and tertiary muon beams from the CERN $450 \mathrm{GeV}$ super proton synchrotron (SPS). Its multi-purpose detector concept allows for a wide range of investigations in hadron physics, with high-precision and high-rate capable tracking, particle identification and calorimetry in both stages of the magnetic spectrometer. The layout of the setup is shown in Fig. 1. The two stages are optimized for low and high momentum particles, respectively, and allow a momentum determination of better than $1 \%$ in a wide range, from about $1 \mathrm{GeV}$ up to the beam momentum in the range of $200 \mathrm{GeV}$.

\section{Primakoff technique for low-energy meson dynamics}

In 1951, Primakoff proposed [2] to make use of the intense electric field in the proximity of nuclei to study interactions with strongly-interacting particles, which appears at highly relativistic motion as a source of quasi-real photons. The original idea concerned the measurement of the $\pi^{0}$ lifetime by photon-photon fusion, but it was later realized that interactions of high-energetic hadrons with the nuclear Coulomb field represent similarly scattering off the quasi-real photon density, and consequently the whole class of such hadron interactions is referred to as Primakoff reactions. The main contribution comes from impact parameters of the incoming particles of a few nuclear radii, where the electric field is still as strong as several $100 \mathrm{kV} / \mathrm{fm}$. This displays how even a small polarisability as it is expected for hadrons, can be measured as a modification of the cross-section for bremsstrahlung emission. The cross-section formula for a Primakoff reaction $\pi^{-} A \rightarrow X^{-} A$ on a nucleus $A$ reads, in one-photon exchange approximation (see e.g. [3]),

$$
\frac{d \sigma}{d s d Q^{2} d \Phi}=\frac{\alpha}{\pi\left(s-m_{\pi}^{2}\right)} \cdot F_{\mathrm{eff}}^{2}\left(Q^{2}\right) \cdot \frac{Q^{2}-Q_{\min }^{2}}{Q^{4}} \cdot \frac{d \sigma_{\pi \gamma}}{d \Phi},
$$


and bases on the factorization into the quasi-real photon density multiplying the cross-section $d \sigma_{\pi \gamma} / d \Phi$ for the real-photon subprocess $\pi^{-} \gamma \rightarrow X^{-}$, with $d \Phi$ being a proper phase space parameterization for the final state of the subprocess. Mandelstam- $s$ is the squared total energy in the $\pi^{-} \gamma$ subsystem, $Q^{2}$ is the momentum transfer to the nucleus $A, \alpha \simeq 1 / 137$ is the fine structure constant, $m_{\pi}$ the rest mass of the charged pion, $Q_{\min }=\left(s-m_{\pi}^{2}\right) / 2 p$ is the minimum momentum transfer for given $s$ and beam momentum $p . F_{\text {eff }}^{2}\left(Q^{2}\right)$ is the form factor of the target nucleus with charge $Z$. It is $F_{\text {eff }}^{2}\left(Q^{2}\right) \approx Z^{2}$ when $Q^{2} \ll 10^{-3} \mathrm{GeV}^{2} / c^{2}$ and in addition $s$ is sufficiently small, such that the longitudinal compontent $Q_{L}$ (given in size approximately by $Q_{\min }$ ) is negligible.

\subsection{Pion polarisability}

For the first process of interest here, the final-state $X^{-}$is again $\pi^{-} \gamma$ such that the involved subprocess is pion Compton scattering $\pi^{-} \gamma \rightarrow \pi^{-} \gamma$. The respective cross-section reads

$$
\begin{aligned}
\frac{d \sigma_{\pi \gamma}}{d \Omega_{c m}}= & \frac{\alpha^{2}\left(s^{2} z_{+}^{2}+m_{\pi}^{4} z_{-}^{2}\right)}{s\left(s z_{+}+m_{\pi}^{2} z_{-}\right)^{2}}-\frac{\alpha m_{\pi}^{3}\left(s-m_{\pi}^{2}\right)^{2}}{4 s^{2}\left(s z_{+}+m_{\pi}^{2} z_{-}\right)} . \\
& \cdot\left(z_{-}^{2}\left(\alpha_{\pi}-\beta_{\pi}\right)+\frac{s^{2}}{m_{\pi}^{4}} z_{+}^{2}\left(\alpha_{\pi}+\beta_{\pi}\right)-\frac{\left(s-m_{\pi}^{2}\right)^{2}}{24 s} z_{-}^{3}\left(\alpha_{2}-\beta_{2}\right)\right),
\end{aligned}
$$

where $z_{ \pm}=1 \pm \cos \theta_{c m}$ and $\theta_{c m}$ is the scattering angle in the $\pi^{-} \gamma$ center-of-momentum system, and the pion structure enters through the electric and magnetic polarisabilities $\alpha_{\pi}$ and $\beta_{\pi}$. The solid angle $d \Omega_{c m}=d \cos \theta_{c m} d \varphi_{c m}$ is the concrete form that the phase space $d \Phi$ of Eq. (1) takes on for the considered two-body reaction at fixed $s$. In the following, the sum $\alpha_{\pi}+\beta_{\pi}$ which is expected to be small, and also the influence of the quadrupole polarisabilities $\alpha_{2}$ and $\beta_{2}$ is neglected. Then, the relative effect of the polarisability $\alpha_{\pi}=-\beta_{\pi}$ on the cross-section, Eq. 1, integrated in the small-momentum transfer region $Q^{2} \leq 10^{-3} \mathrm{GeV}^{2} / c^{2}$ and depending only on the fraction of energy transferred from the incoming pion beam to the emitted photon, $x_{\gamma}=E_{\gamma} / E_{\text {beam }}$, can be simplified into

$$
R=\frac{\sigma\left(x_{\gamma}\right)}{\sigma_{\alpha_{\pi}=0}\left(x_{\gamma}\right)}=1-\frac{3}{2} \cdot \frac{m_{\pi}^{3}}{\alpha} \cdot \frac{x_{\gamma}^{2}}{1-x_{\gamma}} \alpha_{\pi} .
$$

This relation is used to extract the polarisability from the measurement of the photon energy spectrum in the the Primakoff process $\pi^{-} Z \rightarrow \pi^{-} \gamma Z$ on a nucleus with charge $Z$, as it has been done in the first measurement of this kind at Serpukhov [4].

As can be read off Eq. (1), the cross-section for Primakoff reactions is proportional to the square of the nuclei charge $Z^{2}$. Since the competing strong interactions only grow roughly with $Z^{2 / 3}$, one the Primakoff contribution can be enhanced by choosing high- $Z$ targets. For systematic effects discussed later, very heavy targets are also disfavored, and for the experiment described here, a target with medium-heavy nickel nuclei was chosen. Measuring with different target nuclei in the same setup allows to study the $Z$-dependence of the different contributions.

After a pilot run in the year 2004, the data presented in the following have been collected in a two-week beam time in 2009, with significant improvements in the calorimetry and the trigger system which based on the detailed analysis of the 2004 data. One of the conclusions when preparing the data taking in 2009 [6] was that lead is not a favorable target material despite the high nuclear charge $Z$, since the radiative corrections due to multiple photon exchange and screening are large and represent a non-negligible source of systematic uncertainty. Consequently, the measurement was performed with a $4 \mathrm{~mm}$ thick nickel disk 
as nuclear target. The measurement and the extraction of the pion polarisability signal is described in detail in [5]. With the assumptions made for Eq. 3, the result determined from the data taken in 2009 is $\alpha_{\pi}=\left(2.0 \pm 0.6_{\text {stat }} \pm 0.7_{\text {syst }}\right) \cdot 10^{-4} \mathrm{fm}^{3}$. The analysis of the high-statistics data set taken in 2012 is still ongoing, with a focus on the analysis of the chiral anomaly that is presented in the following section.

\subsection{Chiral anomaly}

The process $\pi^{-} \gamma \rightarrow \pi^{-} \pi^{0}$ is dominated by the $\rho(770)$ resonance in the region up to CM energies $\sqrt{s} \lesssim 1.4 \mathrm{GeV}$, however in the threshold region at $\mathrm{CM}$ energies smaller than the $\rho$ mass, there is a contribution from a direct photon-3-pion interaction expected from ChPT, called the chiral anomaly. It has been carefully worked out, including the interference with the $\rho$ resonance, in the theory paper [7].

The process is experimentally very similar to the previously treated reaction with $\pi \gamma$ in the final state, except that here appear the two decay photons from the $\pi^{0}$, and the observed final state is $\pi \gamma \gamma$. Using the four-vectors of the two photons, the peak in the invariant mass spectrum at the $\pi^{0}$ mass is selected, as shown in Fig. 2 (left), for which already the low- $Q^{2}$ region has been selected, in order to ensure photon exchange.

The invariant-mass spectrum of the final state $\pi^{-} \pi^{0}$ for the selected events is shown in Fig. 2 (left). It still contains background from reactions with more particles in the final state, which escape detection, mainly from $\pi^{-} \gamma \rightarrow \pi^{-} \pi^{0} \pi^{0}$ and the corresponding reaction with Pomeron exchange. The latter contributes rather at higher $Q^{2}$, but since one (soft) $\pi^{0}$ escapes detection, the reconstruction of the momentum transfer is distorted and results in a contribution to the selected small- $Q^{2}$ window. This has been carefully investigated, taking the measured part of $\pi^{-} \pi^{0} \pi^{0}$ and using the Monte-Carlo simulation in order to estimate it. Due to the comparatively large cross section for Pomeron exchange, it turns out to be quite significant. The resulting background-corrected invariant mass spectrum is shown in Fig. 3.

The data are fitted with the functional shape according to [7], extended by the addition of a Gaussian-smeared peak that allows to take into account the observed fraction of free kaon decays. Overall, the model fits very well the observed spectrum, and from the fitted region (red curve in Fig. 3) can even be extrapolated to lower and higher masses without systematic deviations (green curves). This encouraging intermediate step can, however, only be correctly interpreted in terms of the chiral anomaly $F_{3 \pi}$ and the radiative coupling of the $\rho$ resonance, once the experimental data are normalized as cross section. This implies the determination of the apparatus acceptance by Monte-Carlo simulation, and the precise knowledge of the luminosity. Similar methods as applied in earlier works of the COMPASS collaboration [8, 9], employing the known free decay of kaon beam component, are in principle applicable, however have not yet been established for reactions with neutral particles in the final state as needed here.

\section{Light-meson spectroscopy}

In similar kinematics as the previously discussed Primakoff reactions, however using a hydrogen target and extending to higher momentum transfer to the recoil protons, the COMPASS collaboration has taken the currently world's largest data set for light-meson spectroscopy with charged and neutral pions and kaons in the final states. Special focus in the analysis of the data taken in the years 2008 and 2009 is made on final states with three pions, since it features the highest number of reconstructed events, about 50 million, and allows most detailed investigations regarding the employed models for the resonance descriptions and the production process in diffractive dissociation. 
COMPASS 2012

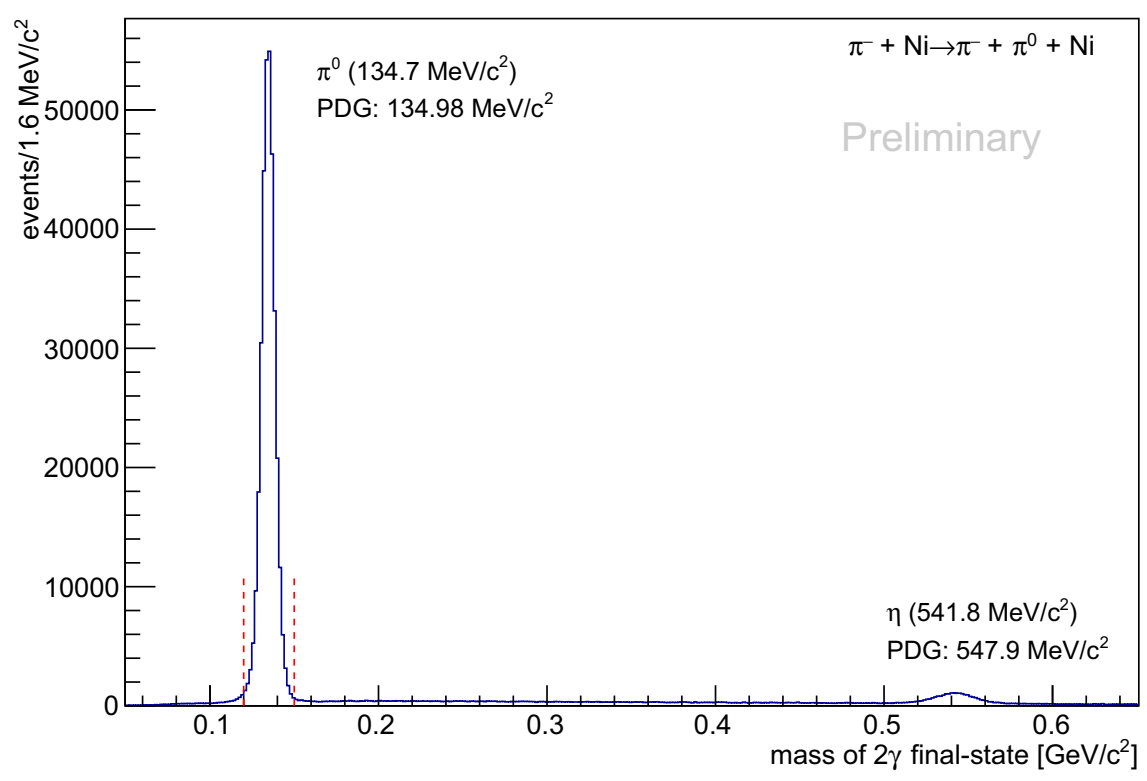

COMPASS 2012

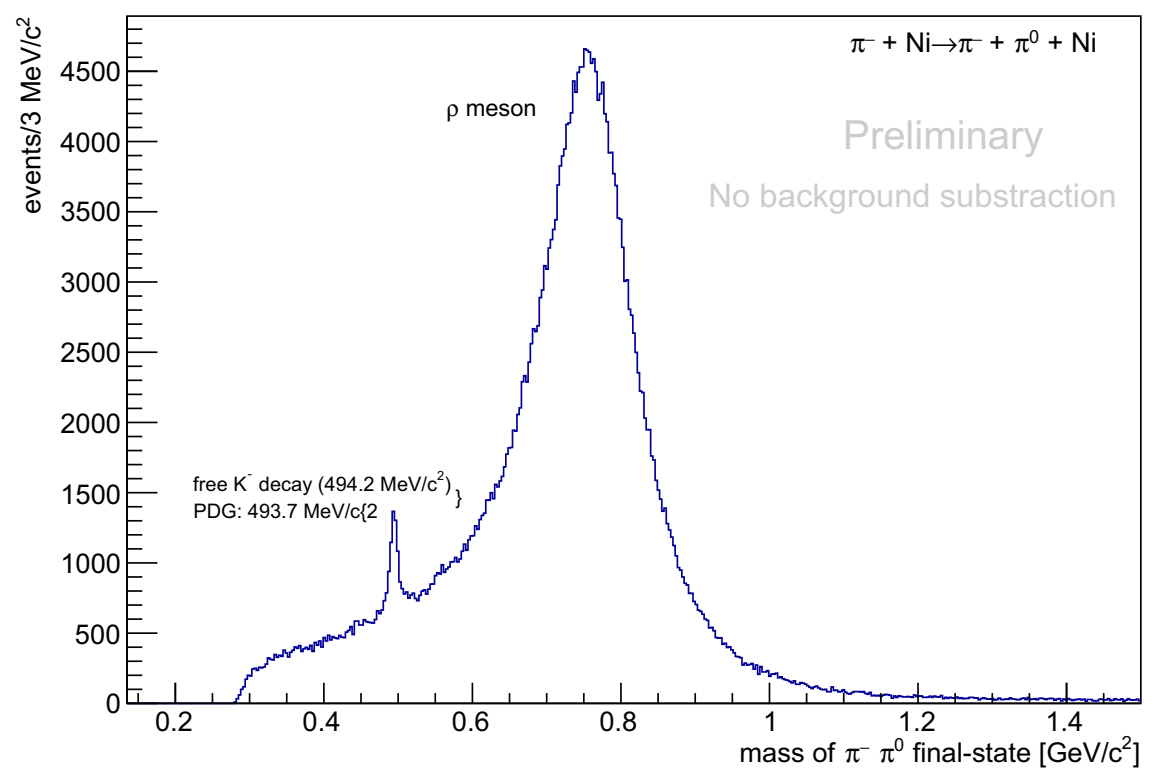

Figure 2. Kinematic selection of the process $\pi^{-} \gamma \rightarrow \pi^{-} \pi^{0}$. Upper plot: Invariant-mass spectrum of the two final-state photons, featuring peaks at the nominal $\pi^{0}$ and $\eta$ masses. The peak corresponding to $\pi^{0}$ production is selected for the further analysis. Lower plot: Invariant mass of the final state $\pi^{-} \pi^{0}$, before background subtraction. The peak of the $\rho(770)$ resonance is salient, the interpretation of the further details of the spectrums can however only be done after background subtraction. Due to the $2.5 \%$ kaon fraction in the incoming beam, a peak from in-flight decays $K^{-} \rightarrow \pi^{-} \pi^{0}$ is also present. 


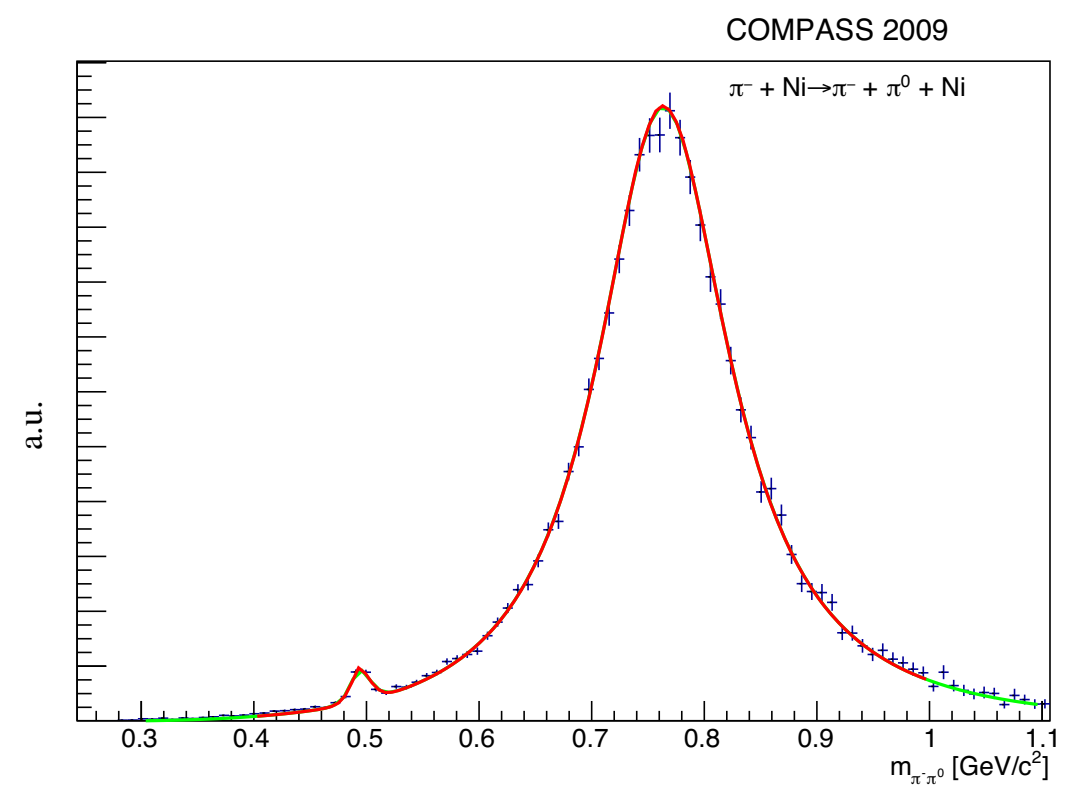

Figure 3. The invariant-mass spectrum for the $\pi^{-} \gamma \rightarrow \pi^{-} \pi^{0}$ process after background subtraction. The shown fit according to [7] is described in the text. The vertical scale is omitted, since the determination of the luminosity of the data is still ongoing.

In order to determine the contributing resonances and their parameters, such as their mass and widths, the data are subject to partial-wave analysis (PWA), assuming two-pion isobar states. A partial wave is defined, on the one hand, by the $J^{P C}$ of the three-pion state and its spin projection $M$ onto the beam axis. The latter leads to a phase modulation of the amplitude, which also determines the reflectivity $\epsilon$ that is linked to the naturality of the exchange mechanism. The reflectivity is expected to be +1 for the diffractive reactions investigated here. On the other hand, the partial wave is given by the isobar description and by the orbital angular momentum $L$ between the isobar and the bachelor pion. In total, a partial wave is specified by $J^{P C} M^{\epsilon}$ [isobar] $\pi L$.

Examplary, three waves with large contribution to the total intensity are shown in Fig. 4. The first column shows the fitted intensity for the strongest $1^{++}$wave, decaying into $\rho \pi$ in an $\mathrm{S}$ wave. A clear feature of this wave, usually referred to as $a_{1}(1260)$ resonance, is the shift of its position depending on the momentum tranfer. It is believed that this is due to interference with the Deck effect, which describes a scattering amplitude without assuming a 3-pion resonance, but only a virtual-pion pole contribution in the exchange trajectory. Work is ongoing in order to take this into account, such that resonance parameters can be extracted more reliably. The presented $a_{2}$ and $a_{4}$ resonances are less concerned by this effect.

In addition, it turns out that the high-statistics COMPASS data set is well adapted to further control the assumptions made in the PWA approach, namely regarding the two-pion resonances. It was shown that by slicing the data set in bins of the two-pion masses, one is able to extract the parameters of the contributing resonances from the three-pion data set. This has been presented in detail in a dedicated contribution to this conference [10]. 

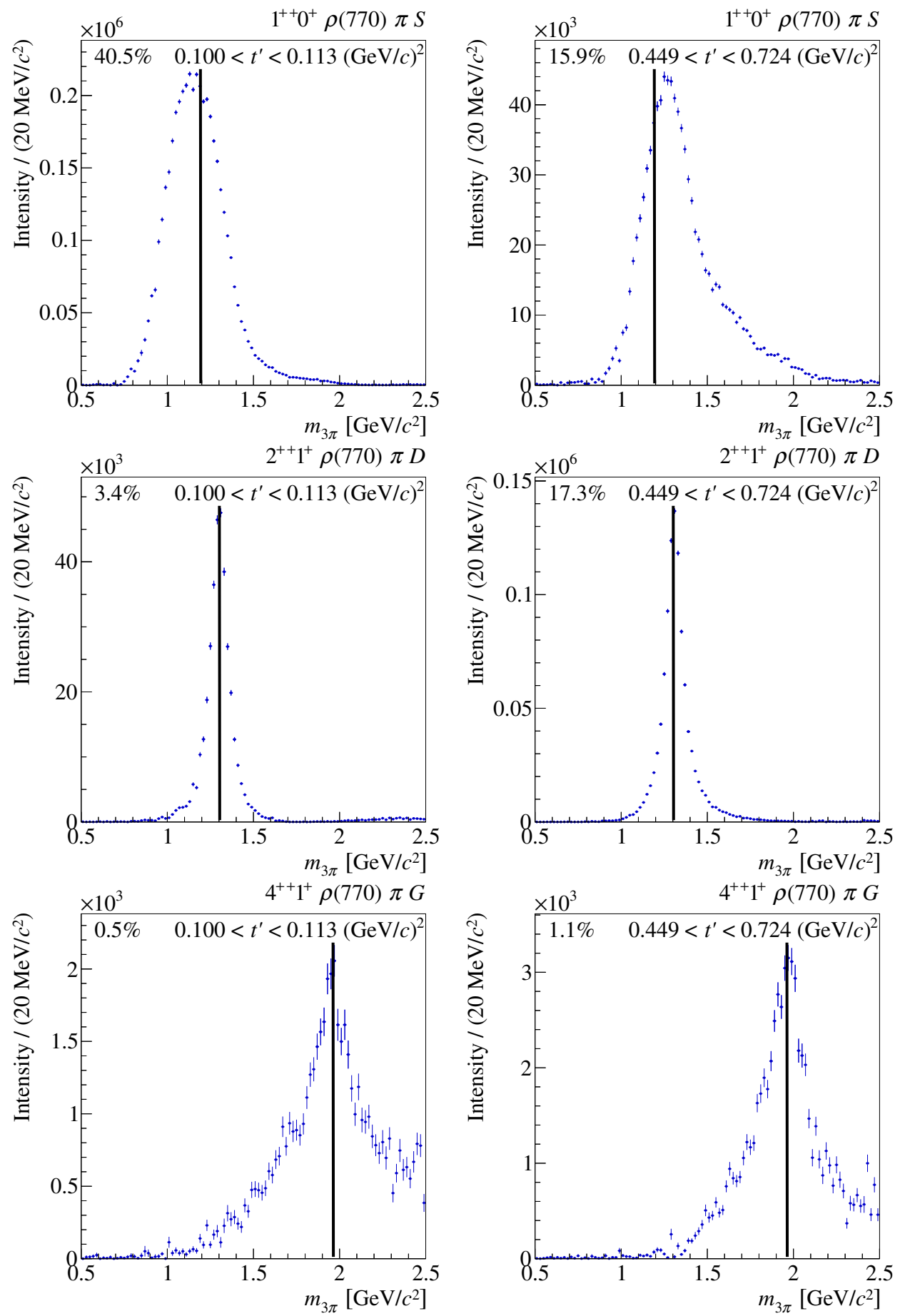

Figure 4. Results for the PWA of 3 charged pions produced in diffractive dissociation at COMPASS. The left column is for the lowest bin in momentum transfer $t^{\prime}$, the right column for the highest $t^{\prime}$ bin. Percentages in the left-upper corner of the graphs indicate the fraction with respect to the total intensity. Further discussion see text. 


\section{References}

[1] S. Grabmüller, PhD thesis, Tech. Univ. München (2012), CERN-THESIS-2012-170

[2] H. Primakoff, Phys. Rev. 81, 899 (1951)

[3] N. Kaiser and J. M. Friedrich, Eur. Phys. J. A36, 181 (2008)

[4] Yu. M. Antipov et al., Phys. Lett. B121, 445 (1983)

[5] C. Adolph et al. [COMPASS Collaboration], Phys. Rev. Lett. 114, 062002 (2015)

[6] N. Kaiser and J. M. Friedrich, Eur. Phys. J. A39, 71 (2009)

[7] M. Hoferichter, B. Kubis, D. Sakkas, Phys. Rev. D86, 116009 (2012)

[8] C. Adolph et al. [COMPASS Collaboration], Phys. Rev. Lett. 108,192001 (2012)

[9] C. Adolph et al. [COMPASS Collaboration], Eur. Phys. J. A50, 79 (2014)

[10] F. Krinner, these proceedings 